A-30-2020

\title{
APLICACIÓN DE LA OXIFERTIRRIGACIÓN PARA OPTIMIZAR LOS RECURSOS HÍDRICOS EN CÍTRICOS, BASADO EN LA APLICACIÓN DE PERÓXIDO DE HIDRÓGENO EN EL AGUA DE RIEGO
}

\author{
Robles García, J.M. (P)(1), Botía Ordaz, P. (1), Olivares Quilez, L. (1), Palazón López, \\ J.A.(1), Navarro Acosta, J.M. (1), Arques Pardo, E.M. (1), Martínez Izquierdo, A.V. (1), \\ Hernández Ballester F.M. (1), Pérez-Pérez, J.G. (1-2)
}

${ }^{1}$ Equipo de Riego y Fisiología del Estrés, Instituto Murciano de Investigación y Desarrollo Agrario y Alimentario (IMIDA), La Alberca (Murcia). E-mail: juanm.robles@carm.es

2 Centro para el Desarrollo de la Agricultura Sostenible (CDAS), Instituto Valenciano de Investigaciones Agrarias (IVIA), Moncada (Valencia). E-mail: perez_juaperb@gva.es

\section{Resumen}

En este trabajo se estudia la respuesta fisiológica y agronómica de la aplicación de la oxifertirrigación química, basada en la aplicación de peróxido de hidrógeno como fuente de oxígeno a nivel radicular en cítricos. El ensayo tuvo lugar durante dos campañas (2018-2019 y 2019-2020) en árboles adultos de mandarino híbrido 'Ortanique' ubicados en una parcela experimental del IMIDA en Torre Pacheco (Murcia). Se establecieron dos tratamientos, un tratamiento 'Control' $\left(0 \mathrm{ppm}\right.$ de $\mathrm{H}_{2} \mathrm{O}_{2}$ ) y otro identificado como 'OXI' (50-100 ppm de $\mathrm{H}_{2} \mathrm{O}_{2}$ durante todo el ciclo de cultivo). Ambos tratamientos recibieron la misma cantidad de agua y de fertilizante. El $\mathrm{H}_{2} \mathrm{O}_{2}$ se aplicó de forma continua con una bomba dosificadora a la red de riego. Los resultados más destacados mostraron que el estado hídrico de los árboles del tratamiento 'OXI' fue muy similar al control. Respecto a los parámetros de intercambio gaseoso, la aplicación de $\mathrm{H}_{2} \mathrm{O}_{2}$ estimuló una mayor apertura estomática en el mes de septiembre de ambas campañas. Sin embargo, las ligeras alteraciones fisiológicas no han supuesto cambios sustanciales en la biometría de la planta. En la segunda campaña, la aplicación de $\mathrm{H}_{2} \mathrm{O}_{2}$ en el riego favoreció la acumulación de $\mathrm{N}, \mathrm{K}$ y Fe en hoja, lo que permitiría reducir la dosis de fertilizante. La respuesta productiva y la eficiencia en el uso del agua no presentaron una clara mejora a la aplicación de $\mathrm{H}_{2} \mathrm{O}_{2}$ en el agua de riego. Los mayores niveles de $\mathrm{N}$ en el tratamiento 'OXI' afectaron negativamente a la calidad del fruto, reduciendo el porcentaje de zumo y aumentando el porcentaje de corteza. En cambio, la aplicación de $\mathrm{H}_{2} \mathrm{O}_{2}$ disminuyó el índice de madurez de la fruta, lo que resulta interesante de cara a retrasar la recolección en variedades tardías.

\section{1- Introducción}

Dentro de los cultivos arbóreos de regadío en la Región de Murcia, los cítricos representan uno de los cultivos más importantes a nivel socioeconómico, siendo el limonero el naranjo y el mandarino los más destacados, con unas producciones en el año 2017 de 555.760 t, 137.345 t y 123.800 t, respectivamente (CARM, 2020).

La climatología de la Región de Murcia es semiárida, y bajo estas condiciones, la viabilidad de las explotaciones agrícolas depende principalmente de la disponibilidad y la gestión de recursos hídricos existentes. Por ese motivo, el manejo del riego y del cultivo dirigido a maximizar la productividad del agua ( $\mathrm{kg}$ de cosecha por unidad de agua de riego aplicada) (Seckle, 1996) adquiere una gran importancia en esta región.

Para un adecuado desarrollo del cultivo, es necesaria la presencia de oxígeno en la disolución del suelo. Las raíces necesitan una concentración de $\mathrm{O}_{2}$ adecuada para funcionar correctamente y satisfacer las necesidades de agua y nutrientes de la parte aérea de la planta 
(Barret-Lennard, 2003; Meek et al., 1983). Sin embargo, determinadas situaciones pueden producir problemas en la planta por falta de $\mathrm{O}_{2}$ en el suelo, que pueden provocar un descenso de la producción en agricultura (Bartholomeus et al., 2008). El uso de aguas de baja calidad con elevada salinidad (Navarro y Pérez Pérez, 2019), la compactación del suelo por el no laboreo (Kumar et al., 2018), el riego en exceso o encharcamiento del suelo por lluvias torrenciales (Morales-Olmedo et al, 2015) son las principales causas que pueden ocasionar este problema. La aplicación de productos que permitan mantener altas concentraciones de oxígeno en la rizosfera durante el ciclo de cultivo, puede asegurar un óptimo funcionamiento radicular. Esto favorecerá el crecimiento y rendimiento del cultivo, mejorando la eficiencia en el uso del agua y de los fertilizantes (Bhattarai et al., 2005).

La oxifertirrigación química es una técnica que consiste en la aplicación de compuestos a base de peróxidos directamente en el agua de riego, que durante el proceso de descomposición liberan oxígeno (Marfa y Gurí, 1999; Urrestarazu y Mazuela, 2005). Con ello se pretende conseguir, por un lado, la desinfección del agua, y por otro, enriquecerla con oxígeno. Debido a su rápida velocidad de reacción y su inocuidad para el medio ambiente, no genera ningún tipo de contaminante por ser biodegradable (Carrasco y Urreztarazu, 2010). Dicha técnica, ha sido utilizada con resultados variables en diversos cultivos hortícolas bajo condiciones de hidroponía. En pimiento, la aplicación de la oxifertirrigación mejoró el rendimiento (Marfa et al., 2005; Urrestarazu y Mazuela 2005). En cambio, sobre el mismo cultivo, pero en perlita, no se encontró ningún efecto sobre la biometría de la planta y el rendimiento al aplicar concentraciones crecientes de $\mathrm{H}_{2} \mathrm{O}_{2}$ (Acuña et al., 2006). En plantación directa en suelo, con textura arcillosa y problemas de falta de oxígeno, la aplicación de $\mathrm{H}_{2} \mathrm{O}_{2}$ en fertirrigación, aumentó la biomasa total y el rendimiento en calabacín (Bhattarai et al., 2004). En cultivos plantados en un suelo similar, pero en maceta, se obtuvo una mejora de la biomasa y de la productividad en soja y principalmente de la productividad en algodón (Bhattarai et al., 2004). Los cítricos necesitan un adecuado contenido de oxígeno en la disolución de suelo para un buen desarrollo radicular que permita suplir a la parte área de los elementos nutricionales necesarios para el cultivo (Labanauskas et al., 1964). Sin embargo, en cítricos, la aplicación de la oxifertirrigación química como herramienta paliativa bajo condiciones de falta de oxígeno está escasamente estudiada.

\section{2- Objetivos}

El objetivo principal del experimento fue evaluar la respuesta fisiológica y agronómica de la aplicación de la oxifertirrigación química, basada en la aplicación de peróxido de hidrógeno, como una herramienta auxiliar para aumentar el contenido de oxígeno a nivel radicular, en árboles adultos de mandarino híbrido 'Ortanique'.

\section{3- Material y Métodos}

El estudio se realizó durante las campañas de 2018-2019 y 2019-2020 en una parcela experimental de cítricos del IMIDA ubicada en Torre Pacheco (Murcia), la cual posee un suelo representativo del campo de Cartagena (textura franco-arcillo-arenosa), con bajo contenido en materia orgánica $(0,53 \%)$ y con un sistema de mantenimiento del suelo de no laboreo. La parcela tiene una superficie de 1 ha y el material vegetal utilizado fueron árboles de 25 años de edad de mandarino híbrido 'Ortanique' (Citrus sinensis (L.) Osb. x Citrus reticulata Blanco) injertados sobre citrange 'Carrizo' (Citrus sinensis Osb. x Poncirus trifoliata (L.) Raf.) con un marco de plantación de $4 \times 3 \mathrm{~m}$. El sistema de riego constó de una línea portagoteros por fila de árboles (renovada al inicio del ensayo), con tres goteros autocompensantes NETAFIM (4 $\mathrm{L} \mathrm{h}^{-1}$ ) por árbol separados $0,75 \mathrm{~m}$ de distancia. La programación del riego se realizó semanalmente, utilizando la evapotranspiración de referencia (ETo) calculada con el método de Penman-Monteih, obtenida a partir de los datos de una estación agroclimática perteneciente al Sistema de Información Agraria de Murcia (SIAM) ubicada en la propia parcela experimental. La dosis de agua de riego se determinó mediante la ecuación: 


$$
\mathrm{ETc}=\mathrm{ETO} \times \mathrm{Kc}
$$

donde la ETo viene en $\mathrm{mm}$ y $\mathrm{Kc}$ es el coeficiente de cultivo proporcionado por el SIAM para variedades de mandarino tardías. Ambos tratamientos se recibieron la misma dosis de riego (100\% de la ETc) y la misma cantidad de fertilizante. El diseño experimental fue de bloques al azar, con tres repeticiones por bloque y cuatro bloques por tratamiento. Se establecieron dos tratamientos, un tratamiento 'Control' ( $0 \mathrm{ppm}$ de $\mathrm{H}_{2} \mathrm{O}_{2}$ ) y otro identificado como 'OXI' (50100 ppm de $\mathrm{H}_{2} \mathrm{O}_{2}$ durante todo el ciclo de cultivo). En el tratamiento 'OXl' el $\mathrm{H}_{2} \mathrm{O}_{2}$ se aplicó de forma continua con una bomba dosificadora digital de membrana a la red de riego.

Para el seguimiento del ensayo se realizaron controles, tanto en el gotero como en el cabezal de riego, del contenido de $\mathrm{O}_{2}$ disuelto en agua, con un equipo portátil multifunción (HATCH mod. HQ 40d). El estado hídrico del cultivo se evaluó mediante la medida del potencial hídrico de tallo al mediodía $\left(\Psi_{\text {tallo }}\right)$ utilizando una cámara de presión (modelo 3000 ; Soil Moisture Equipment Corp.). Las medidas de intercambio gaseoso se realizaron quincenalmente, en días soleados, entre las 08:30 y las 10:00 horas con un sistema portátil de fotosíntesis (Li-6400, Li-COR, Lincoln, Nebraska, EE.UU.) obteniendo los valores de fotosíntesis $\left(A_{\mathrm{CO} 2}\right)$, conductancia estomática $\left(g_{s}\right)$ y transpiración $\left(E_{h o j a}\right)$. En ambos años se realizó un control del estado nutricional del cultivo en el mes de noviembre, para ello se tomaron muestras de 25 hojas por árbol, completamente maduras, de la brotación de primavera y sin frutos. La variación del perímetro del tronco se determinó mediante el uso de dendrómetros de crecimiento de acero inoxidable $( \pm 0,1 \mathrm{~mm})(\mathrm{mod}$. DB-20, Environmental Measuring System). La tasa de crecimiento absoluto del tronco (AGR) se calculó según PérezPérez et al., 2014. Al finalizar la segunda campaña, se realizó la poda del cultivo determinando el peso fresco de poda $\left(\mathrm{kg}\right.$ árbol $\left.{ }^{-1}\right)$ mediante una báscula digital $( \pm 0.05 \mathrm{~kg})$. En el mes de febrero de ambos años de ensayo, se controló la producción, el número de frutos y se determinó el peso medio del fruto de cada árbol. Se calculó la productividad del agua como la relación entre la cosecha y el agua de riego aplicada $\left(\mathrm{kg} \mathrm{m}^{-3}\right)$. En el momento de la cosecha, se tomó una muestra de nueve frutos por árbol (12 por tratamiento) para analizar posteriormente la calidad del fruto. Los parámetros físico-químicos de calidad del fruto evaluados fueron: diámetro del fruto $(\mathrm{mm})$, Índice de color externo $\left(\mathrm{IC}_{\text {ext }}\right)$, espesor de corteza (mm), porcentaje de zumo, corteza y pulpa, sólidos solubles totales (SST; ${ }^{\circ}$ Brix), acidez valorable (AT; $\mathrm{g} \mathrm{L}^{-1}$ ) e índice de madurez (IM) (Pérez-Pérez et al. 2014). El análisis estadístico consistió en un ANOVA unifactorial con dos tratamientos y doce repeticiones por tratamiento (tres árboles monitorizados por tratamiento y bloque) despreciando los árboles situados en los extremos. Para ello se utilizó el paquete estadístico Statsgraphics Centurión XV (StatPoint Technologies Inc.). Para la separación de medias se aplicó el test de rango múltiple de Duncan $(P<0.05)$.

\section{4- Resultados y Discusión}

Durante la primera campaña de 2018-2019, la aplicación de 100 ppm de $\mathrm{H}_{2} \mathrm{O}_{2}$ ocasionó problemas puntuales de obturación de goteros, como consecuencia del arrastre de partículas orgánicas de las paredes de las tuberías secundarias y terciarias que no fueron renovadas. Esto supuso una reducción del agua de riego aplicada del $7 \%$ en el tratamiento 'OXI' respecto al tratamiento control (tabla 1). Después de 48 días (desde 24/04/2018 hasta el 11/06/2018), se procedió a reducir la dosis de $\mathrm{H}_{2} \mathrm{O}_{2}$ a 50 ppm para evitar este problema durante el resto del ciclo de cultivo (figura 1A). En la campaña 2019-2020, se continuó manteniendo el tratamiento 'OXI' con 50 ppm de $\mathrm{H}_{2} \mathrm{O}_{2}$ hasta el 20/05/2019 (en total 343 días desde el 11/06/2018) y después de constatar la no existencia de obturaciones en goteros se subió a 100 ppm hasta el final del ciclo de cultivo (figura 1B). Durante esta segunda campaña, la cantidad de agua aplicada fue similar entre ambos tratamientos (tabla 1).

El contenido de $\mathrm{O}_{2}$ disuelto en el agua de riego a la salida del gotero durante 2019 fue significativamente más elevado en el tratamiento 'OXI' (> $20 \mathrm{ppm})$ frente al tratamiento control (9-10 ppm) en el momento del muestreo (0 horas) (Figura 2). Ese mayor contenido de $\mathrm{O}_{2}$ en el agua de riego en el tratamiento 'OXI' se mantuvo, al menos, hasta las 7 horas desde la 
toma de muestra, para posteriormente, presentar valores similares al control (Figura 2). Esto permitiría mantener unos valores de $\mathrm{O}_{2}$ en la disolución de suelo superiores al tratamiento control, que podría provocar una mejora en el desarrollo radicular del cultivo en el tratamiento 'OXI' (Bhattarai, et al., 2010). Las diferencias en los niveles de $\mathrm{O}_{2}$ disuelto en el agua de riego no supusieron una clara mejora del estado hídrico de la planta. En 2018 el tratamiento 'OXI' presentó valores significativamente más bajos de $\Psi_{\text {tallo }}$ que el control a primeros de junio, consecuencia de las obturaciones de goteros indicadas anteriormente que redujeron la cantidad de agua aplicada (figura $1 \mathrm{~A}$ ). Posteriormente, después ajustar la dosis de $\mathrm{H}_{2} \mathrm{O}_{2}$ a 50 $\mathrm{ppm}$, disminuyeron considerablemente las obturaciones en goteros, y el tratamiento OXI mostró valores significativamente superiores al control a primeros de julio para la misma cantidad de agua aplicada (figura 1A). Aunque fue de manera puntual y las diferencias significativas no se mantuvieron en el tiempo, dichas diferencias se produjeron en un momento de máxima demanda climática. En 2019 no se encontraron diferencias en el $\Psi_{\text {tallo }}$ entre los tratamientos estudiados a lo largo de la campaña (figura 1B).

Tabla 1. Valores de evapotrasnpiración, precipitación y agua aplicada para cada uno de los tratamientos (Control y 'OXI') durante las campañas de 2018-2019 y 2019-2020

\begin{tabular}{|c|c|c|}
\hline & Campaña 2018-2019 & Campaña 2019-2020 \\
\hline ETo $(\mathrm{mm})$ & 1245 & 1140 \\
\hline Precipitación (mm) & 245 & 594 \\
\hline \multicolumn{3}{|l|}{ Agua Aplicada ( $\mathrm{mm}$ ) } \\
\hline Tratamiento Control & 588 & 528 \\
\hline Tratamiento OXI & 548 & 528 \\
\hline
\end{tabular}

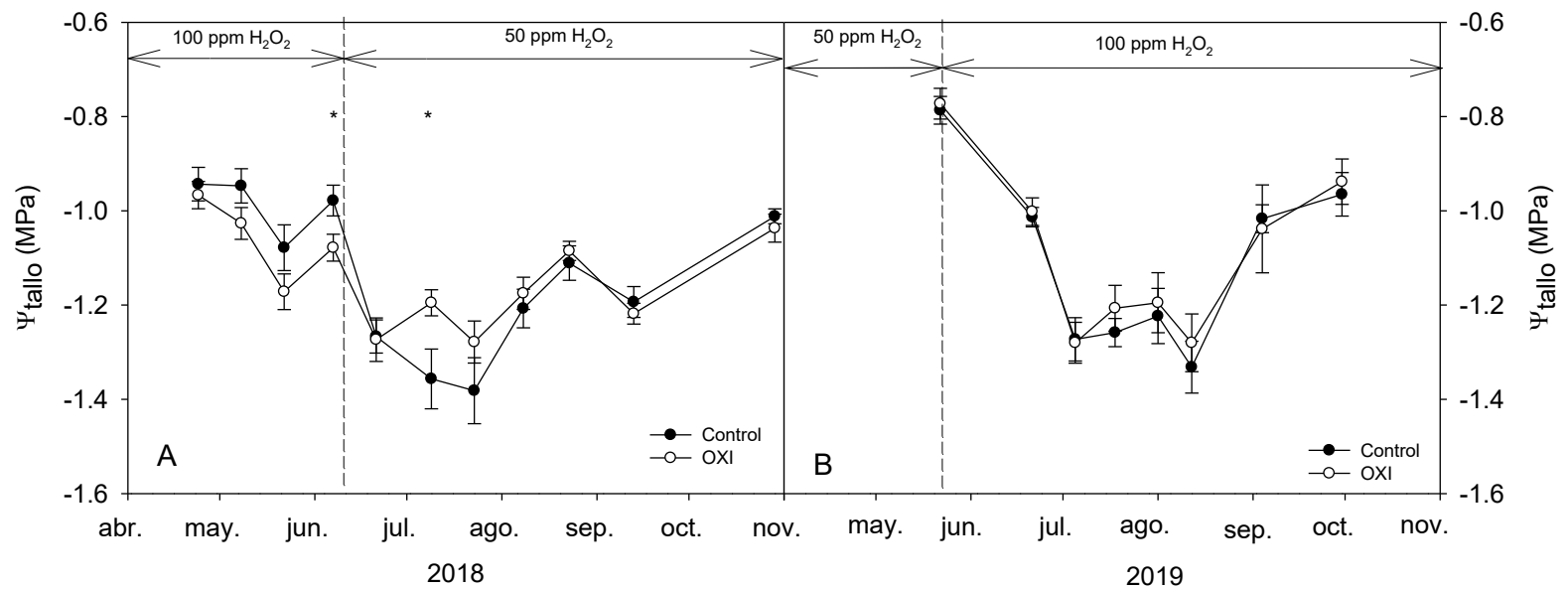

Figura 1. Evolución estacional de la aplicación de $\mathrm{H}_{2} \mathrm{O}_{2}$ y del potencial hídrico de tallo al mediodía ( $\left.\Psi_{\text {tallo }}\right)$ de los tratamientos Control y 'OXI' en árboles de mandarino 'Ortanique' en 2018 (A) y 2019 (B). 


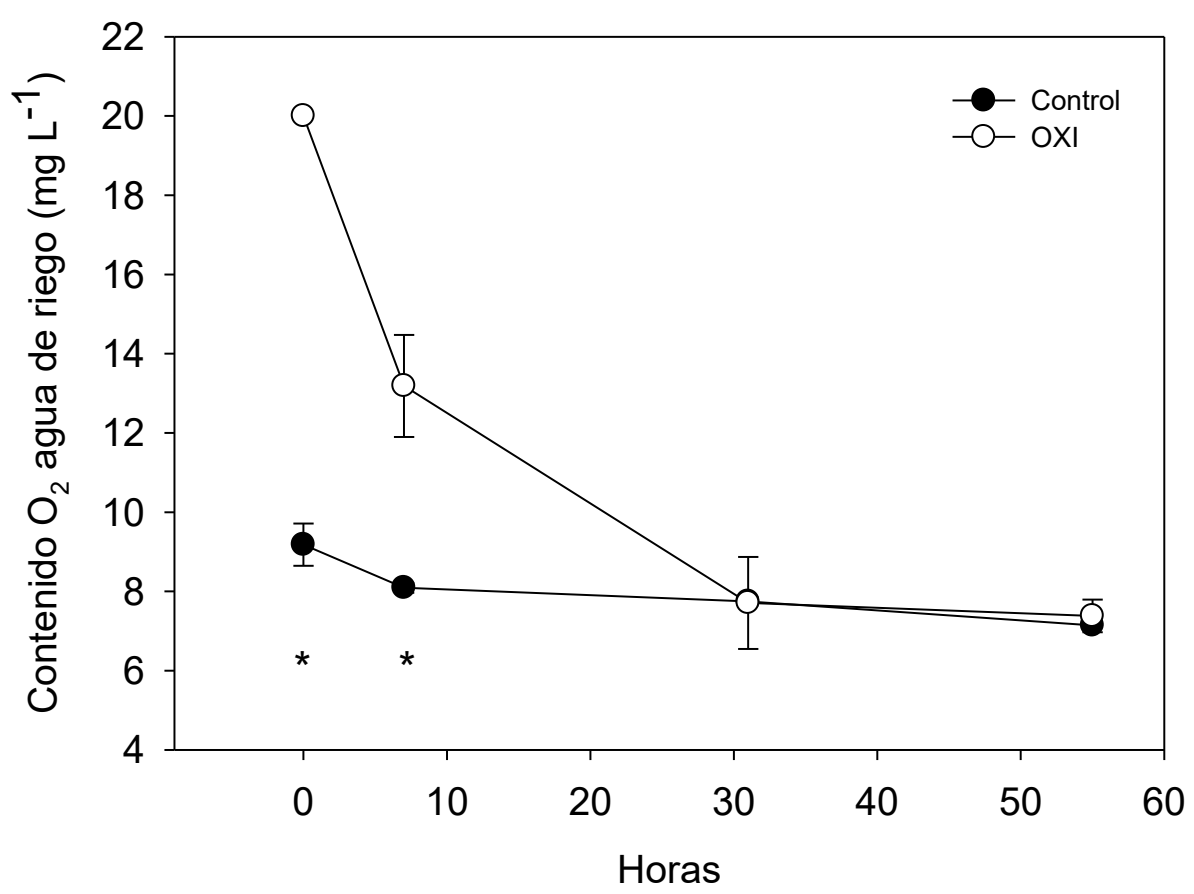

Figura 2. Evolución del contenido de $\mathrm{O}_{2}$ del agua de riego tomado a la salida del gotero analizado a las $0,7,31$ y $55 \mathrm{~h}$ de los tratamientos control y OXI en árboles de mandarino 'Ortanique' en el mes de junio de 2019.

La aplicación de $\mathrm{H}_{2} \mathrm{O}_{2}$ influyó positivamente sobre los parámetros de intercambio gaseoso. El tratamiento 'OXI' no aumentó los valores de $A_{\mathrm{co} 2}$, pero presentó valores significativamente superiores de $g_{s}$ y $E_{\text {hoja }}$ en el mes de septiembre de ambas campañas (figura 3). Dicho aumento, estuvo entorno al $29 \%$ en los valores de $g_{s}$ y del $20 \%$ en $E_{\text {hoja. }}$ Una respuesta similar se ha obtenido en cultivos hortícolas como el sorgo, y que podría estar relacionada con un mayor desarrollo radicular por la aplicación de $\mathrm{H}_{2} \mathrm{O}_{2}$ (Bhattarai et al., 2004). Sin embargo, pese a la mejora de los parámetros de intercambio gaseoso en el tratamiento 'OXl', no alteró el crecimiento vegetativo del cultivo. El experimento se inició en árboles con similar diámetro de tronco (tabla 2). La AGR no mostró diferencias significativas entre tratamientos en ambas campañas (tabla 2). Además, la poda realizada al finalizar la última campaña fue similar entre los tratamientos estudiados (tabla 2). Similar respuesta se ha obtenido en cultivos hortícolas bajo hidroponía, donde la aplicación de $\mathrm{H}_{2} \mathrm{O}_{2}$ no ha producido cambios significativos en la biometría de la planta (Acuña et al., 2006; Soto-Bravo, 2015).

Tabla $\mathbf{n}^{\circ} 2$. Diámetro del tronco al inicio del experimento, Tasa de crecimiento absoluto 20182019 (AGR 2018-2019) y 2019-2020 (AGR $2019-2020)$, Peso fresco de poda de (2019-2020) de los tratamientos control y OXI en árboles de mandarino 'Ortanique'.

\begin{tabular}{lcccc}
\hline & $\begin{array}{c}\text { Diámetro tronco } \\
(\mathbf{c m})\end{array}$ & $\begin{array}{c}\mathbf{A G R}_{\mathbf{2 0 1 8 - 2 0 1 9}} \\
(\mathbf{m m})\end{array}$ & $\begin{array}{c}\mathbf{A G R}_{\mathbf{2 0 1 9 - 2 0 2 0}} \\
(\mathbf{m m})\end{array}$ & $\begin{array}{c}\text { Peso de poda } \\
\left.\mathbf{( k g ~ a ́ r b o l ~}^{-1}\right)\end{array}$ \\
\hline Control & 18,8 & 10,6 & 10,4 & 12,2 \\
Oxi & 20,0 & 8,0 & 11,1 & 14,6 \\
Anova & & & & $\mathrm{ns}$ \\
\hline Signif. & $\mathrm{ns}$ & $\mathrm{ns}$ & & $\mathrm{ns}$ \\
\hline ns = no significativo. & & &
\end{tabular}

ns = no significativo.

DOI:10.31428/10317/8685 


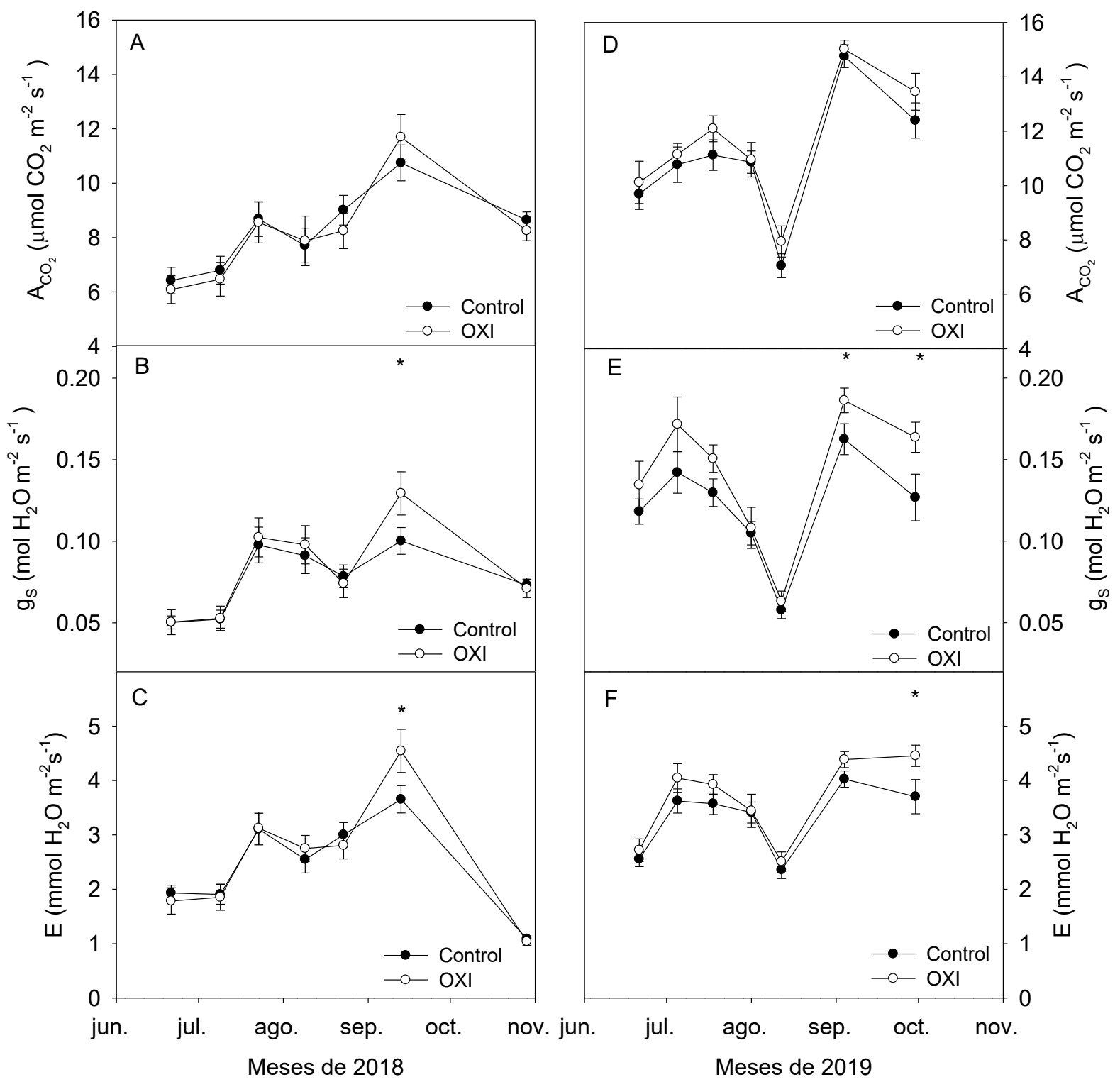

Figura 3. Evolución estacional de la fotosíntesis $\left(A_{\mathrm{co} 2}\right)$, conductancia estomática $\left(g_{s}\right)$ y de la tasa de transpiración ( $E_{\mathrm{Hoja}}$ ) de los tratamientos control y OXI en árboles de mandarino 'Ortanique' durante los años 2018 (A, B y C) y 2019 (D, E y F).

En relación con el estado nutricional del cultivo, en la primera campaña, el tratamiento 'OXI' no modificó la composición mineral en hoja (tabla 3). En cambio, en la segunda campaña, la aplicación de $\mathrm{H}_{2} \mathrm{O}_{2}$ favoreció la acumulación de elementos minerales en la hoja, principalmente $\mathrm{N}, \mathrm{K}$ y Fe (tabla 3 ). El aumento de la temperatura durante los meses de verano puede limitar los niveles de $\mathrm{O}_{2}$ de la solución de suelo (Bhattarai, et al., 2010) reduciendo la absorción de nutrientes en el cultivo, particularmente $\mathrm{N}$ y Fe (Morard et al., 2004), por lo que la aplicación de $\mathrm{H}_{2} \mathrm{O}_{2}$ podría evitar ese problema. De hecho, los árboles del tratamiento 'OXl' acumularon significativamente un $4 \%$ más en el contenido de $\mathrm{N}$, un $26 \%$ en el de $\mathrm{K}$ y un $10 \%$ en el contenido de Fe (tabla 3). De acuerdo con las normas de producción integrada en cítricos de la Región de Murcia (Borm, 2012), los valores obtenidos de $\mathrm{N}$ en el tratamiento 'OXI' estarían próximos a valores altos, mientras que los del tratamiento control se encuentran dentro del intervalo de valores normales. Respecto al $\mathrm{K}$, los valores son muy bajos para ambos tratamientos y el Fe se encontraría en el intervalo de valores normales. En relación con el $\mathrm{N}$, esa mayor acumulación en el tratamiento 'OXl' sugiere una mayor eficiencia en el uso de ese fertilizante ya que se aplicó la misma cantidad de $\mathrm{N}$ en ambos tratamientos. Esto podría ser 
interesante en aquellas zonas vulnerables por contaminación de nitratos, donde la aplicación de $\mathrm{H}_{2} \mathrm{O}_{2}$ podría favorecer una reducción en la aplicación de $\mathrm{N}$.

Tabla 3. Contenido mineral en hoja de mandarino 'Ortanique' para cada uno de los tratamientos estudiados (control y OXI) en noviembre de 2018 y de 2019.

\begin{tabular}{|c|c|c|c|c|c|c|c|c|c|c|c|}
\hline 2018 & $\begin{array}{l}\mathbf{N} \\
\% \\
\end{array}$ & $\begin{array}{l}\mathbf{P} \\
\%\end{array}$ & $\begin{array}{l}\mathrm{K} \\
\% \\
\end{array}$ & $\begin{array}{l}\mathrm{Ca} \\
\%\end{array}$ & $\begin{array}{c}\mathrm{Mg} \\
\%\end{array}$ & $\begin{array}{c}\mathrm{Na} \\
\%\end{array}$ & $\begin{array}{c}\mathrm{Fe} \\
\mathrm{ppm}\end{array}$ & $\begin{array}{c}\mathrm{Cu} \\
\mathrm{ppm}\end{array}$ & $\begin{array}{c}\text { Mn } \\
\text { ppm }\end{array}$ & $\begin{array}{c}\mathrm{Zn} \\
\mathrm{ppm}\end{array}$ & $\begin{array}{c}\text { B } \\
\text { ppm }\end{array}$ \\
\hline Control & 2,61 & 0,15 & 0,33 & 4,49 & 0,62 & 0,18 & 79 & 16 & 35 & 34 & 271 \\
\hline $\begin{array}{l}\text { Oxi } \\
\text { Anova }\end{array}$ & 2,62 & 0,15 & 0,36 & 4,22 & 0,60 & 0,24 & 84 & 18 & 38 & 37 & 272 \\
\hline Signif. & ns & ns & ns & ns & ns & ns & ns & ns & ns & ns & ns \\
\hline \multicolumn{12}{|l|}{2019} \\
\hline Control & $2,77 b$ & 0,13 & $0,35 \mathrm{~b}$ & 4,24 & 0,52 & 0,13 & $65 \mathrm{~b}$ & 23 & 23 & 19 & 275 \\
\hline $\begin{array}{l}\text { Oxi } \\
\text { Anova }\end{array}$ & $2,87 \mathrm{a}$ & 0,13 & $0,44 \mathrm{a}$ & 4,28 & 0,50 & 0,17 & $72 a$ & 24 & 23 & 21 & 281 \\
\hline Signif. & * & ns & * & ns & $\mathrm{ns}$ & ns & * & ns & ns & ns & ns \\
\hline
\end{tabular}

${ }^{*} \mathrm{y}$ ' $\mathrm{ns}$ ' indican diferencias significativas a $\mathrm{P}<0.05$ y no significativas respectivamente.

Aunque la aplicación de $\mathrm{H}_{2} \mathrm{O}_{2}$ aumentó la $\mathrm{g}_{\mathrm{s}}$ y la $\mathrm{E}_{\text {hoja }}$ en determinados momentos de ciclo de cultivo y mejoro la acumulación en hoja de determinados elementos minerales en la última campaña, no mejoró significativamente la respuesta productiva del cultivo ni la eficiencia en el uso del agua (EUA) (tabla 4). Destacar que, en la última campaña, se observó una tendencia a la mejora de dichos parámetros, donde la cosecha y la eficiencia en el uso del agua aumentaron en el tratamiento 'OXl' en un $20 \%$, pero sin diferencias significativas (tabla 4). Al igual que se ha observado anteriormente con la biometría del cultivo, la aplicación de $\mathrm{H}_{2} \mathrm{O}_{2}$, puede llegar a no presentar una mejora de la productividad del cultivo. En pimiento y tomate bajo condiciones de hidroponía, la mejora de la oxigenación del agua no supuso una clara mejora del rendimiento del cultivo (Acuña et al., 2006; Soto-Bravo, 2015).

Tabla 4. Respuesta productiva de cada uno de los tratamientos estudiados (control y OXI) y eficiencia productiva en el uso del agua en mandarino 'Ortanique'.

\begin{tabular}{|c|c|c|c|c|}
\hline 2018-2019 & $\mathrm{n}^{0}$ frutos árbol $^{-1}$ & $\mathrm{Kg}$ árbol-1 & Peso medio (g) & EUA $\left(\mathrm{Kg} \mathrm{m}^{-3}\right)$ \\
\hline Control & 416 & 77,9 & 197,9 & 11,0 \\
\hline $\begin{array}{l}\text { Oxi } \\
\text { Anova }\end{array}$ & 421 & 79,3 & 199,0 & 12,1 \\
\hline Signif. & ns & ns & ns & ns \\
\hline 2019-2020 & & & & \\
\hline $\begin{array}{l}\text { Control } \\
\text { Oxi } \\
\text { Anova }\end{array}$ & $\begin{array}{l}270 \\
336\end{array}$ & $\begin{array}{l}53,0 \\
64,6\end{array}$ & $\begin{array}{l}211,8 \\
198,2\end{array}$ & $\begin{array}{c}8,4 \\
10,2\end{array}$ \\
\hline Signif. & ns & ns & ns & ns \\
\hline
\end{tabular}

ns = no significativo.

Por lo contrario, la mejora en la oxigenación del agua de riego alteró la calidad del fruto (tabla 5). En la primera campaña, el tratamiento 'OXI' modificó principalmente los parámetros físicos de calidad del fruto. Aumentó el espesor de corteza y mejoró la coloración del fruto, pero redujo el porcentaje de zumo respecto al tratamiento control. En la segunda campaña, donde se aumentó la dosis de $\mathrm{H}_{2} \mathrm{O}_{2}$, la reducción significativa del porcentaje de zumo fue mayor, próxima al $11 \%$, consecuencia de un aumento del porcentaje de corteza por un valor similar (tabla 5). Esto es un factor negativo de calidad respecto al tratamiento control y puede 
estar relacionado con la mayor acumulación de $\mathrm{N}$ en el tratamiento 'OXl'. Los mayores niveles de $\mathrm{N}$ en el tratamiento 'OXI' pueden implicar un mayor porcentaje de corteza y con ello una reducción del porcentaje de zumo (Legaz et al., 2000). Respecto a los parámetros químicos de calidad del fruto, en esta campaña se produjo un aumento de la acidez en un $16 \%$ que redujo el índice de madurez (I.M.) en el tratamiento 'OXI' (tabla 5). Esto puede resultar interesante de cara a retrasar la cosecha, siendo de interés en variedades de recolección tardía, como puede ser en este caso la mandarina 'Ortanique'.

Tabla 5. Principales parámetros de calidad del fruto en arboles de mandarino 'Ortanique'.

\begin{tabular}{|c|c|c|c|c|c|c|c|c|c|}
\hline 2018-2019 & $\begin{array}{c}\text { Diámetro } \\
\text { (mm) }\end{array}$ & $\mathbf{I C}_{\text {ext }}$ & $\begin{array}{c}\text { Esp. } \\
\text { corteza } \\
(\mathrm{mm})\end{array}$ & $\begin{array}{c}\% \\
\text { Zumo }\end{array}$ & $\begin{array}{c}\% \\
\text { Pulpa }\end{array}$ & $\begin{array}{c}\% \\
\text { Corteza }\end{array}$ & $\begin{array}{c}\text { SST } \\
\text { ('brix) }\end{array}$ & $\begin{array}{c}\text { Acidez } \\
\text { (g/L) }\end{array}$ & I.M. \\
\hline Control & 80,5 & $19,8 \mathrm{~b}$ & $3,0 \mathrm{~b}$ & $54,8 \mathrm{a}$ & 3,4 & $41,6 \mathrm{~b}$ & 12,4 & 11,4 & 10,0 \\
\hline $\begin{array}{l}\text { OXI } \\
\text { Anova }\end{array}$ & 81,6 & $20,5 \mathrm{a}$ & $3,2 \mathrm{a}$ & $52,7 \mathrm{~b}$ & 3,3 & $43,5 a$ & 12,2 & 12,6 & 9,7 \\
\hline Signf. & ns & * & * & * & $\mathrm{ns}$ & * & $\mathrm{ns}$ & $\mathrm{ns}$ & $\mathrm{ns}$ \\
\hline $2019-2020$ & & & & & & & & & \\
\hline Control & 82,0 & 20,1 & 3,3 & $48,5 \mathrm{a}$ & 2,8 & $47,7 \mathrm{~b}$ & 11,1 & $12,7 \mathrm{~b}$ & $8,1 \mathrm{a}$ \\
\hline $\begin{array}{l}\text { OXI } \\
\text { Anova }\end{array}$ & 80,8 & 19,6 & 3,1 & $43,3 b$ & 2,7 & $53,0 \mathrm{a}$ & 10,9 & 14,7 a & $7,4 \mathrm{~b}$ \\
\hline Signf. & ns & ns & ns & $* *$ & ns & $* *$ & ns & * & ** \\
\hline
\end{tabular}

\section{5- Conclusiones}

La aplicación de la oxifertirrigación mejoró la respuesta fisiológica del cultivo, aumentando los valores de la $g_{s}$ y la $E_{\text {hoja, }}$ al final de la fase II desarrollo del fruto. Sin embargo, no se ha observado una respuesta clara a mejorar la productividad del cultivo y del agua aplicada. Se ha puesto de manifiesto que la aplicación de $\mathrm{H}_{2} \mathrm{O}_{2}$ favorece la acumulación de $\mathrm{N}$ en hoja, lo que permitiría reducir la dosis en el plan de abonado. Respecto a la calidad del fruto, pese a reducir el porcentaje de zumo, favorece un retraso en la maduración del fruto lo que resulta interesante en variedades de recolección tardía. El seguimiento del ensayo en años posteriores permitirá confirmar los resultados preliminares obtenidos.

\section{6- Agradecimientos}

Este trabajo ha sido cofinanciado por el Proyecto de Investigación FEDER 14-20-24 dentro del programa operativo de la Región de Murcia 2014-2020 cofinanciado en un 80\% por el Fondo Europeo de Desarrollo Regional y por el Subprograma Estatal de Incorporación Ramón y Cajal (RYC-2015-17726), en el marco del Plan Estatal de Investigación Científica y Técnica y de Innovación del Ministerio de Economía y Competitividad.

\section{7- Bibliografía}

Acuña, R., Bonachela, S., y Magán, J. (2006). Respuesta de un cultivo de pimiento en sustrato de perlita a la mejora de la oxigenación del medio radicular. Acta de Horticultura, 46, 91-95.

Barret-Lennard, E.G. (2003) The interaction between water logging and salinity in higher plants: causes, consequences and implications. Plant and soil, 253, 35-54

Bartholomeus, R.P., Witte, J.P.M., van Bodegom, P.M., van Dam, J.C., Aerts, R. (2008). Critical soil conditions for oxygen stress to plant roots: substituting the Feddes-function by a process-based model. Journal Hydrology, 360, 147-165. 
Bhattarai, S., Huber, S., and Midmore. D. (2004) Aerated subsurface irrigation water gives growth and yield benefits to zucchini, vegetable soybean and cotton in heavy clay soils. Annals of Applied Biology 144, 285-298.

Bhattarai, S.P., Midmore,D.J., and Su, N. (2010) Sustainable Irrigation to Balance Supply of Soil Water, Oxygen, Nutrients and Agro-Chemicals. E. Lichtfouse (ed.), Biodiversity, Biofuels, Agroforestry and Conservation Agriculture Sustainable Agriculture Reviews 5, DOI 10.1007/978-90-481-9513-8_9,

Bhattarai, S., Su, N and Midmore, D. (2005). Oxygation unlocks yield potentials of crops in oxygen-limited soil environments. Advances in Agronomy. 88, 313-377.

Boletín Oficial de la Región de Murcia (BORM) núm. 100, (2012). Orden de 24 de abril de 2012, de la Consejería de Agricultura y Agua por la que se regulan las normas técnicas de producción integrada en el cultivo de cítricos. Consejería de Agricultura y Agua, pp. 1837718402.

CARM, (2020). Comunidad Autónoma Región de Murcia. Portal estadístico de la Región de Murcia. http://econet.carm.es/. Acceso marzo 2020.

Carrasco, G., and Urrestarazu, M. (2010). Green chemistry in protected horticulture: The use of peroxyacetic acid as a sustainable strategy. International of Journal of Molecular Sciences 11, 1999-2009.

Huang, B.R., Johnson, J.W., Nesmith, D.S., Bridges, D.C. (1994) Root and shoot growth of wheat genotypes in response to hypoxia and subsequent resumption of aeration. Crop Science 34, 1538-1544

Kumar V, Butter T.S., Samanta, A., Singh, G., Kumar, M., Dhotra, B., Yadab N.K and Choudhary R.S. (2018) Soil compaction and their management in farming systems: A review. International Journal of Chemical Studies 6, 2302-2313

Labanauskas, C. F., Stolzy, L. H., Klotz, L. J. and DeWolfe, T. A. (1964) Adequate soiloxygen supplies increase nutrient concentrations in citrus seedlings. California Agriculture 18 (10), 13-14.

Legaz, F., Bañuls, J., Primo-Millo, E. (2000). Influencia del abonado en la calidad del fruto. Levante Agrícola. 350, 12-17.

Marfa, O., y Gurí, S (1999). Física de sustratos y oxigenación del medio radicular. En: cultivo sin suelo II: Curso superior de especialización. Coord. por M. Fernández; Cuadrado, I. IFAPA y Caja Rural de Almería, Almería, ESP. p. 93-106.

Marfa, O., Cáceres, R and Gurí, S. (2005) Oxyfertigation: a new technique for soilless culture under Mediterranean conditions. Acta Horticulturae. 697,65-72.

Meek, B.D., Ehlig, C.F., Stolzy, L.H., Graham, L.E. (1983) Forrow and trickle irrigation: Effects on soil oxygen and ethylene and tomato yield. Soil Science Society of American Journal, 47, 631-635

Morales-Olmedo, M., Ortiz, M., Selles, G. (2015). Effects of transient soil waterlogging and its importance for rootstock selection. Chilean Journal of. Agricultural. Research 75, 4556

Morard, P., Lacoste, L., Silvestre, J. (2004). Effect of oxygen deficiency on mineral nutrition of excised tomato roots. Journal of Plant Nutrition 27, 651-661

Navarro, J.M. y Pérez Pérez, J.M. (2019). Oxifertirrigación química en el cultivo de plantas de pimiento en condiciones salinas. Agrícola Vergel, 416, 11-17.

Pérez-Pérez, J.G., Robles, J.M., Botía, P. (2014). Effects of deficit irrigation in different fruit growth stages on 'Star Ruby' grapefruit trees in semi-arid conditions. Agricultural Water Management, 133, 44-54

Seckler, D. (1996). The new era of water resources management: from "dry" to "wet" water savings. Colombo, Sri Lanka: International Irrigation Management Institute (IIMI). iii, 17p.

Soto-Bravo, F. (2015). Oxifertirrigación química mediante riego en tomate hidropónico cultivado en invernadero. Agronomía mesoamericana, 26, 277-289

Urrestarazu, M., and Mazuela, P. (2005). Effect of slow-release oxygen supply by fertigation on horticultural crops under soilless culture. Science Horticulturae. 106, 484-490. 